\title{
A Numerical Approach to a Nonlinear and Degenerate Parabolic Problem by Regularization Scheme
}

\author{
Haitao Cao \\ Department of Mathematics and Physics, Changzhou Campus, Hohai University, Changzhou, China \\ Email: 20104007004@suda.edu.cn
}

Received October 2013

\begin{abstract}
In this work we propose a numerical scheme for a nonlinear and degenerate parabolic problem having application in petroleum reservoir and groundwater aquifer simulation. The degeneracy of the equation includes both locally fast and slow diffusion (i.e. the diffusion coefficients may explode or vanish in some point). The main difficulty is that the true solution is typically lacking in regularity. Our numerical approach includes a regularization step and a standard discretization procedure by means of $\mathrm{CO}$-piecewise linear finite elements in space and backward-differences in time. Within this frame work, we analyze the accuracy of the scheme by using an integral test function and obtain several error estimates in suitable norms.
\end{abstract}

Keywords

Nonlinear Degenerate Parabolic Equation, Finite Element Method, Regularization, Implicit Scheme

\section{Introduction}

Denoting $\Omega \subset R^{n} \quad(n \geq 1)$ as the domain occupied by the porous medium with Lipschitz boundary and $(0, T](0$ $<T<+\infty$ ) as the time interval. In this work we propose a numerical method to the following nonlinear and degenerate parabolic equation.

$$
\partial_{\mathrm{t}} \mathrm{b}(\mathrm{u})-\operatorname{div}(\mathrm{A}(\mathrm{x}) \nabla \mathrm{u}+\mathrm{g}(\mathrm{x}, \mathrm{b}(\mathrm{u})))=0,(\mathrm{x}, \mathrm{t}) \in \Omega \times(0, \mathrm{~T}]
$$

with the boundary and initial conditions

$$
\mathrm{u}=0 \text { on } \partial \Omega \times(0, \mathrm{~T}], \mathrm{u}(\mathrm{x}, 0)=\mathrm{u} 0 \geq 0 \text { in } \Omega,
$$

where the function $b(s)$ is uniformly bounded, continuous and strictly increasing in $s$. But the degeneracy conditions, $b^{\prime}(s)=0$ and $b^{\prime}(s)=+\infty$ for some point, are included. It means that the equation includes both locally fast and slow diffusion.

Throughout this paper we assume, without loss of generality, that $b^{\prime}(s)=0, s \rightarrow+\infty$ and $b^{\prime}(0+)=+\infty$. Thus the problem (1.1)-(1.2) often used to describe the flows in porous media, infiltration and multi-phase change see [1]- 
[11] etc. The existence of variational solution of (1.1)-(1.2) has been studied in [5] [6] etc. Here we point out that the Equation (1.1) is usually obtained after using the Kirchhoff transformation. Such problems can be investigated through a parabolic regularization of function $b(u)$ or by perturbing the boundary and initial data such that the corresponding solutions do not take the degenerate points. In the past several decades, there are numerous papers about discussing this problem. For example, in [4] [7], the authors consider a rather general class of singular parabolic problems; two-phase Stefan problem and porous medium equation are included. By using a regularization scheme and a parabolic duality technique, a fully discrete numerical approach is proposed and analyzed. In [8] [9], Richards' equation is analyzed by a numerical approach also consisting in a regularization procedure and discretization by means of $\mathrm{C} 0$-piecewise linear finite elements (or mixed finite elements) in space and backward-differences in time. In [10], basing on the maximum principle, the authors considered the porous medium equation by perturbing the boundary and initial data to overcome the degeneracy. On the other hand, there are many other papers dealing with such problem by some (linear) relaxation schemes or imposing some suitable conditions to deal with the degeneracy and nonlinearity [11] 1213].

However, to our knowledge, most of papers have considered one of the degenerate cases, i.e. $b^{\prime}(s)=0$ for some point but $b$ is Lipchtize continuous (such as the Richards' equation) or $b^{\prime}(s)>0$ but discontinuous at one point 0 (such as Stefan problem or porous-medium equation). In our paper, we will deal with the two degenerate cases simultaneously and present the error estimates of several unknowns by using an integral test function. Due to the singularity and degeneracy of $b$, solutions of (1.1) may not be classical; therefore they must be understood in the sense of distribution [5]. The proposed method in the paper, we first replace $b$ by a regular function $b \epsilon$ (whose derivative is bounded by two values depending on regularized parameter $\epsilon>0$ ). The second step is a standard discretization procedure by means of $C 0$-piecewise linear finite elements in space and backward-differences in time. Within this frame work, we analyze the accuracy of the scheme by using an integral test function and obtain several error estimates in suitable norms.

The layout of the paper is as follows. In Section 2, we give out the numerical formulation and main result.

In Section 3, we will prove a prior estimate and the main result of our paper.

Notations: $\Omega_{\mathrm{T}}=\Omega \times(0, \mathrm{~T}), \mathrm{L}^{2}\left(0, \mathrm{~T} ; \mathrm{H}_{0}^{1}(\Omega), \mathrm{H}^{-1}(\Omega)\right.$ is de dual space of $H_{0}^{1}$. Other Soblev space can be referred to [13]. From now on, $C$ will denote a generic positive constant which is independent of $\epsilon$.

\section{Problem Setting and Main Result}

For the problem (1.1)-(1.2), we make the following hypotheses upon the data.

\section{Assumption:}

(H1) The function $b$ mapping $[0,+\infty] \mapsto[0,1]$ is continuous and strictly increasing. And $b^{\prime}(s)=0, s \rightarrow+\infty$, $b^{\prime}(0)=+\infty$.

(H2) $A=(A(x))_{i j}(1 \leq i, j \leq n): R^{n} \mapsto M^{n}$ is continuous and satisfies

$$
\exists 0<\lambda_{1} \leq \lambda_{2}, \lambda_{1}|\xi|^{2} \leq A \xi \cdot \xi \leq \lambda_{2}|\xi|^{2}, \xi \in R^{n} .
$$

(H3) $\mathrm{g}=(\mathrm{g}(\mathrm{x}, \mathrm{s}))_{\mathrm{i}}(1 \leq \mathrm{i} \leq \mathrm{n}): \mathrm{R}^{\mathrm{n}} \times \mathrm{R} \rightarrow \mathrm{R}^{\mathrm{n}}$ is continuous in $s$ and fulfill the following identities:

$$
|g(y, b(u))-g(y, b(v))|^{2} \leq C|b(u)-b(v)||u-v| .
$$

(H4) $\mathrm{u} 0 \in \mathrm{L}^{\infty}(\Omega) \cap H^{2}(\Omega)$.

Remark 2.1. Due to the maximum principle, the solution of the problem (1.1)-(1.2) is great or equal to zero [9] [10]. In physical model, the function b(s) usually presents the enthalpy of Stefan model or denotes the reduced saturation of fluid in porous media.

According to Alt and Luckhaus [5], we have at least that $\partial_{\mathrm{t}} \mathrm{b}(\mathrm{u}) \in \mathrm{L}^{2}\left(0, \mathrm{~T} ; \mathrm{H}^{-1}(\Omega)\right), \mathrm{u} \in \mathrm{L}^{2}\left(0, \mathrm{~T} ; \mathrm{H}_{0}^{1}(\Omega)\right)$.

Because $b$ is uniformly bounded, therefore we conclude that $\mathrm{b}(\mathrm{u}) \in \mathrm{C}^{0}\left(0, \mathrm{~T} ; \mathrm{H}^{-1}(\Omega)\right)$. This gives us $b(u(\cdot, t))$ point wise for every $t \in(0, T]$.

Let $T_{H}$ be a decomposition of $\Omega$ into a regular conforming finite element mesh with maximal element diameter $H$. Denote $\Omega=\cup_{K \in T_{H}} K$. Then we define finite element space $X_{H}$ as:

$$
X_{H}=\left\{\chi \in C^{0}(\Omega) ; \chi \text { is linear for all } K \text { and }\left.\chi\right|_{\partial \Omega}=0\right\} .
$$

Let $N$ be an integer, $\tau=T / N, t_{i}=i \tau$. Our numerical method reads as: find $v_{i}^{H} \in X_{H}, \eta_{i}=b_{\varepsilon}\left(v_{i}^{H}\right) \quad(i=1, \cdots$, $N$ ), for all $\chi \in X_{H}$ 


$$
\left(\frac{\eta_{i}-\eta_{i-1}}{\tau}, \chi\right)+\left(A(x) \nabla v_{i}^{H}+g\left(x, \eta_{i}\right), \nabla \chi\right)=0, v_{0}^{H}=I_{H} u_{0},
$$

where $I_{H}$ is the $C 0$-piecewise linear interpolant operator to $X_{H}$ and satisfies: for $u \in H^{2}(\Omega)$

$$
\left\|I_{H} u-u\right\|_{L^{2}(\Omega)} \leq C H^{s}\|u\|_{H^{s}(\Omega)}, 1 \leq s \leq 2,
$$

and $b_{\varepsilon}(s)$ is defined as

$$
b_{\varepsilon}(s)=\left\{\begin{array}{l}
\frac{\varepsilon}{b^{-1}(\varepsilon)} s, s \in\left[0, b^{-1}(\varepsilon)\right] \\
b(s)+\varepsilon s, s \in\left(b^{-1}(\varepsilon),+\infty\right]
\end{array} .\right.
$$

In the above terms, $\epsilon$ is small parameter. It is easy to get

$$
\left|b(s)-b_{\varepsilon}(s)\right| \leq \max \{\varepsilon, \varepsilon|s|\}, \varepsilon \leq b_{\varepsilon}^{\prime}(s) \leq \frac{\varepsilon}{b^{-1}(\varepsilon)},
$$

The Equation (2.3) is a general nonlinear elliptic problem. It can be numerically solved by relaxation iteration scheme or to apply a linearization scheme first. We point out that the regularization (2.5) of a degenerate problem is not necessary in implementing numerical analysis with suitable assumptions [11].

Our main result of the numerical approach is present as the following:

Theorem 2.2. Let $u, \theta=b(u)$ and $v_{k}^{H}$ be the solution of (1.1) and (2.3) respectively. Suppose (H1)-(H4) hold, then there exist

$$
\int_{0}^{t_{n}}\left(\theta-b\left(v_{k}^{H}\right), u-v_{k}^{H}\right)_{\Omega}+\left\|\sum_{k=1}^{n} \int_{t_{k-1}}^{t_{k}} \nabla\left(u-v_{k}^{H}\right)\right\|_{L^{2}(\Omega)}^{2}+\sum_{k=1}^{n}\left\|\int_{t_{k-1}}^{t_{k}} \nabla\left(u-v_{k}^{H}\right)\right\|_{L^{2}(\Omega)}^{2} \leq C\left(\tau+H^{2}+\varepsilon^{2}\right)
$$

where the constant $\mathrm{C}$ is independent of $\tau, \epsilon, \mathrm{H}$.

Remark 2.3. Due to the lacking regularity of solution, the result of Theorem 2.2 is not optimal with respect to time discretization. On the other hand, if $\mathrm{b}^{\prime}$ is positive and bounded, we can get the classical results.

\section{Proof of Main Result}

Because of the lacking regularity, the solution of (1.1)-(1.2) must be understood in terms of distributions, as proposed in [5]. Firstly, we formulate the weak solution of (1.1)-(1.2) as follows:

Definition3.1. We say that $u$ is a weak solution of problem (1.1)-(1.2), if it satisfies the following two identities:

(1) $\mathrm{b}(\mathrm{u}) \in \mathrm{L}^{2}\left(\Omega_{\mathrm{T}}\right)$ and $\partial_{\mathrm{t}} \mathrm{b}(\mathrm{u}) \in \mathrm{L}^{2}\left(0, \mathrm{~T} ; \mathrm{H}^{-1}(\Omega)\right)$ with

$$
\int_{\Omega_{T}} \partial_{\mathrm{t}} \mathrm{b}(\mathrm{u}) \varphi \mathrm{dxdt}+\int_{\Omega_{T}}\left(\mathrm{~b}(\mathrm{u}(\mathrm{t}))-\mathrm{b}\left(\mathrm{u}^{0}\right)\right) \partial_{\mathrm{t}} \varphi \mathrm{dxdt}=0,
$$

for every $\varphi \in L^{2}\left(0, T ; H^{1}(\Omega)\right) \cap H^{1}\left(0, T ; L^{2}(\Omega)\right)$ with $\varphi(T)=0$.

(2) For $\varphi \in \mathrm{L}^{2}\left(0, \mathrm{~T} ; \mathrm{H}^{1}(\Omega)\right)$

$$
\int_{\Omega_{T}} \partial_{\mathrm{t}} \mathrm{b}(\mathrm{u}) \varphi \mathrm{dxdt}+\int_{\Omega_{T}}(A(x) \nabla u+g(x, b(u))) \cdot \nabla \varphi \mathrm{dxdt}=0,
$$

According to [5], it follows that

Lemma3.2. Assuming (H1)-(H4) hold, if u is a solution of (3.7)-(3.8), we have

$$
\max _{t \in[0, T]}\|B(u(t))\|_{L^{1}(\Omega)}+\left\|\partial_{t} b(u)\right\|_{L^{2}\left(0, T ; H^{-1}(\Omega)\right)}+\|\nabla u\|_{L^{2}\left(\Omega_{T}\right)} \leq C
$$

where $B(u(t))=u(t) b(u(t))-\int_{0}^{u(t)} b(s) d s$.

For the discrete scheme (2.3), we also have

Lemma3.3. Assuming (H1)-(H4) hold, if for all $i=1 \cdots N, v_{i}^{H}$ solves problem (2.3), for any $0<k<N$, we have

$$
\int_{\Omega} B\left(b_{\varepsilon}\left(v_{k}^{H}\right)\right) d x+\tau \sum_{i=1}^{k}\left\|\nabla v_{i}^{H}\right\|_{L^{2}(\Omega)}^{2} \leq C .
$$


Proof: Let $\chi=v_{i}^{H}$ in (2.3), we have

$$
\left(\eta_{i}-\eta_{i-1}, v_{i}^{H}\right)_{\Omega}+\tau\left(A(x) \nabla v_{i}^{H}+g(x) \eta_{i}, \nabla v_{i}^{H}\right)_{\Omega}=0
$$

Summing $i=1 \cdots k$, we get

$$
\left(\eta_{k}, v_{k}^{H}\right)_{\Omega}-\left(\eta_{0}, v_{0}^{H}\right)_{\Omega}-\sum_{i=1}^{k}\left(\eta_{i-1}, v_{i}^{H}-v_{i-1}^{H}\right)_{\Omega}+\sum_{i=1}^{k} \tau\left(A(x) \nabla v_{i}^{H}+g\left(x, \eta_{i}\right), \nabla v_{i}^{H}\right)_{\Omega}=0
$$

Denote the above terms by $I_{1}+I_{2}+I_{3}=0$.

$$
\begin{aligned}
& I_{1} \geq\left(\eta_{k}, v_{k}^{H}\right)_{\Omega}-\left(\eta_{0}, v_{0}^{H}\right)_{\Omega}-\sum_{i=1}^{k} \int_{\Omega} \int_{v_{i-1}^{H}}^{v_{i}^{H}} b_{\varepsilon}(s) d s d x=\int_{\Omega} B\left(b_{\varepsilon}\left(v_{k}^{H}\right)\right) d x+\int_{\Omega} B\left(b_{\varepsilon}\left(v_{0}^{H}\right)\right) d x, \\
& I_{2} \geq \tau \sum_{i=1}^{k}\left\|\nabla v_{i}^{H}\right\|_{L^{2}(\Omega)}^{2}, \quad\left|I_{3}\right| \leq C+\frac{\tau}{2} \sum_{i=1}^{k}\left\|\nabla v_{i}^{H}\right\|_{L^{2}(\Omega)}^{2} .
\end{aligned}
$$

Combining all the above terms, we get the conclusion.

Proof of Theorem 2.2: Let $\theta=b(u(t))$, we integrate (1.1) over time from $t_{i-1}$ to $t_{i}$, for every $\chi \in X_{H}$, to get

$$
\left(\theta_{i}-\theta_{i-1}, \chi\right)_{\Omega}+\left(\int_{t_{i-1}}^{t_{i}} A(x) \nabla u d t, \nabla \chi\right)_{\Omega}+\left(\int_{t_{i-1}}^{t_{i}} g(\theta) d t, \nabla \chi\right)_{\Omega}=0,
$$

Subtract (2.3) from (3.9), we get

$$
\left(\theta_{i}-\eta_{i}-\left(\theta_{i-1}-\eta_{i-1}\right), \chi\right)_{\Omega}+\left(\int_{t_{i-1}}^{t_{i}} A(x) \nabla\left(u-v_{i}^{H}\right) d t, \nabla \chi\right)_{\Omega}+\left(\int_{t_{i-1}}^{t_{i}} g(\theta)-g\left(\eta_{i}\right) d t, \nabla \chi\right)_{\Omega}=0
$$

Summing the equality from $i=1$ to $k$, we obtain

$$
\left(\theta_{k}-\eta_{k}-\left(\theta_{0}-\eta_{0}\right), \chi\right)_{\Omega}+\sum_{i=1}^{k}\left(\int_{t_{i-1}}^{t_{i}} A(x) \nabla\left(u-v_{i}^{H}\right) d t, \nabla \chi\right)_{\Omega}+\sum_{i=1}^{k}\left(\int_{t_{i-1}}^{t_{i}} g(\theta)-g\left(\eta_{i}\right) d t, \nabla \chi\right)_{\Omega}=0 .
$$

Summing $k$ from 1 to $n$ again, we get

$$
\sum_{k=1}^{n}\left(\theta_{k}-\eta_{k}-\left(\theta_{0}-\eta_{0}\right), \chi\right)_{\Omega}+\sum_{k=1}^{n} \sum_{i=1}^{k}\left(\int_{t_{i-1}}^{t_{i}} A(x) \nabla\left(u-v_{i}^{H}\right) d t, \nabla \chi\right)_{\Omega}+\sum_{k=1}^{n} \sum_{i=1}^{k}\left(\int_{t_{i-1}}^{t_{i}} g(\theta)-g\left(\eta_{i}\right) d t, \nabla \chi\right)_{\Omega}=0 .
$$

Setting $\chi=\int_{t_{k-1}}^{t_{k}} I_{H} u-v_{k}^{H} d t \in X_{H}$ and $e_{u}=u-I_{H} u, \theta_{0}=b\left(u_{0}\right), \eta_{0}=b_{\varepsilon}\left(I_{H} u_{0}\right)$, to gives

$$
\begin{aligned}
& \sum_{k=1}^{n}\left(\theta_{k}-\eta_{k}-\left(\theta_{0}-\eta_{0}\right), \int_{t_{k-1}}^{t_{k}}\left(u-v_{k}^{H}\right) d t\right)_{\Omega}+\sum_{k=1}^{n} \sum_{i=1}^{k}\left(\int_{t_{i-1}}^{t_{i}} A(x) \nabla\left(u-v_{i}^{H}\right) d t, \int_{t_{k-1}}^{t_{k}} \nabla\left(u-v_{k}^{H}\right) d t\right)_{\Omega} \\
& +\sum_{k=1}^{n} \sum_{i=1}^{k}\left(\int_{t_{i-1}}^{t_{i}} g(\theta)-g\left(\eta_{i}\right) d t, \int_{t_{k-1}}^{t_{k}} \nabla\left(u-v_{k}^{H}\right) d t\right)_{\Omega} \\
& =\sum_{k=1}^{n}\left(\theta_{k}-\eta_{k}-\left(\theta_{0}-\eta_{0}\right), \int_{t_{k-1}}^{t_{k}} e_{u} d t\right)_{\Omega}+\sum_{k=1}^{n} \sum_{i=1}^{k}\left(\int_{t_{i-1}}^{t_{i}} A(x) \nabla\left(u-v_{i}^{H}\right) d t, \int_{t_{k-1}}^{t_{k}} e_{u} d t\right)_{\Omega} \\
& +\sum_{k=1}^{n} \sum_{i=1}^{k}\left(\int_{t_{i-1}}^{t_{i}} g(\theta)-g\left(\eta_{i}\right) d t, \int_{t_{k-1}}^{t_{k}} e_{u} d t\right)_{\Omega}
\end{aligned}
$$

Denoting the above formulation by $I_{1}+I_{2}+I_{3}=I_{4}+I_{5}+I_{6}$, we will deal with each terms in the following.

$$
\begin{aligned}
I_{1} & =\sum_{k=1}^{n} \int_{t_{k-1}}^{k}\left(\theta_{k}-\eta_{k}, u-v_{k}^{H}\right)_{\Omega} d t-\sum_{k=1}^{n} \int_{t_{k-1}}^{k}\left(\theta_{0}-\eta_{0}, u-v_{k}^{H}\right)_{\Omega} d t=I_{11}+I_{12}, \\
I_{11} & =\sum_{k=1}^{n} \int_{t_{k-1}}^{k}\left(\theta-b\left(v_{k}^{H}\right), u-v_{k}^{H}\right)_{\Omega} d t+\sum_{k=1}^{n} \int_{t_{k-1}}^{k}\left(b\left(v_{k}^{H}\right)-\eta_{k}, u-v_{k}^{H}\right)_{\Omega} d t+\sum_{k=1}^{n} \int_{t_{k-1}}^{k}\left(\int_{t}^{t_{k}} \partial_{s} \theta d s, u-v_{k}^{H}\right)_{\Omega} d t \\
& =I_{111}+I_{112}+I_{113},
\end{aligned}
$$

It is easy to see that $I_{111} \geq 0$. Use (2.6) and Lemma 3.3, it follows that 


$$
\left|I_{112}\right| \leq C(\delta) \sum_{k=1}^{n} \varepsilon^{2} \tau\left\|v_{k}^{H}\right\|_{L^{2}(\Omega)}^{2}+\delta \sum_{k=1}^{n}\left\|\int_{t_{k-1}}^{t_{k}} \nabla\left(u-v_{k}^{H}\right)\right\|_{L^{2}(\Omega)}^{2} \leq C(\delta) \varepsilon^{2}+\delta \sum_{k=1}^{n}\left\|\int_{t_{k-1}}^{t_{k}} \nabla\left(u-v_{k}^{H}\right)\right\|_{L^{2}(\Omega)}^{2}
$$

Considering that $\partial_{\mathrm{t}} \mathrm{b}(\mathrm{u}) \in \mathrm{L}^{2}\left(0, \mathrm{~T} ; \mathrm{H}^{-1}(\Omega)\right), \mathrm{u} \in \mathrm{L}^{2}\left(0, \mathrm{~T} ; \mathrm{H}_{0}^{1}(\Omega)\right)$, we get

$$
\left|I_{113}\right| \leq \tau \int_{t_{k-1}}^{t_{k}}\left\|\partial_{t} \theta\right\|_{H^{-1}(\Omega)}\left\|u-v_{k}^{H}\right\|_{H_{0}^{1}(\Omega)} d t \leq C \tau .
$$

So we get

$$
I_{11} \geq \int_{t_{0}}^{t_{n}}\left(\theta-b\left(v_{k}^{H}\right), u-v_{k}^{H}\right)_{\Omega} d t-C(\delta)\left(\varepsilon^{2}+\tau\right)-\delta \sum_{k=1}^{n}\left\|\int_{t_{k-1}}^{t_{k}} \nabla\left(u-v_{k}^{H}\right)\right\|_{L^{2}(\Omega)}^{2} .
$$

Because $\left|\theta_{0}-\eta_{0}\right| \leq\left|b\left(u_{0}\right)-b_{\varepsilon}\left(u_{0}\right)\right|+\left|b_{\varepsilon}\left(u_{0}\right)-b_{\varepsilon}\left(I_{H} u_{0}\right)\right|$, we have

$$
\begin{aligned}
& \left|I_{12}\right| \leq C(\delta) \sum_{k=1}^{n} \tau\left(\varepsilon^{2}\left\|u_{0}\right\|_{L^{2}(\Omega)}^{2}+H^{4}\left\|u_{0}\right\|_{H^{2}(\Omega)}^{2}\right)+\delta \sum_{k=1}^{n}\left\|\int_{t_{k-1}}^{t_{k}} \nabla\left(u-v_{k}^{H}\right)\right\|_{L^{2}(\Omega)}^{2} \\
& \leq C(\delta)\left(\varepsilon^{2}+H^{4}\right)+\delta \sum_{k=1}^{n}\left\|\int_{t_{k-1}}^{t_{k}} \nabla\left(u-v_{k}^{H}\right)\right\|_{L^{2}(\Omega)}^{2} .
\end{aligned}
$$

Using the following equality

$$
2 \sum_{k=1}^{n} a_{k}\left(\sum_{i=1}^{k} a_{i}\right)=\left(\sum_{k=1}^{n} a_{k}\right)^{2}+\sum_{k=1}^{n} a_{k}^{2}
$$

we can get

$$
\begin{aligned}
& I_{2}=\frac{1}{2}\left\|\sum_{k=1}^{n} A(x) \int_{t_{k-1}}^{t_{k}} \nabla\left(u-v_{k}^{H}\right) d t\right\|_{L^{2}(\Omega)}^{2}+\frac{1}{2} \sum_{k=1}^{n}\left\|A(x) \int_{t_{k-1}}^{t_{k}} \nabla\left(u-v_{k}^{H}\right) d t\right\|_{L^{2}(\Omega)}^{2} \\
& \geq C\left(\left\|\sum_{k=1}^{n} \int_{t_{k-1}}^{t_{k}} \nabla\left(u-v_{k}^{H}\right) d t\right\|_{L^{2}(\Omega)}^{2}+\sum_{k=1}^{n}\left\|\int_{t_{k-1}}^{t_{k}} \nabla\left(u-v_{k}^{H}\right) d t\right\|_{L^{2}(\Omega)}^{2}\right) .
\end{aligned}
$$

For the term $I_{3}$, noticing $\eta_{i}=b_{\varepsilon}\left(v_{i}^{H}\right)$, we use (H3), Cauchy inequality and the fact (2.6) to get

$$
\begin{aligned}
& I_{3}=\sum_{k=1}^{n} \sum_{i=1}^{k}\left(\int_{t_{i-1}}^{t_{i}} g(\theta)-g\left(b\left(v_{i}^{H}\right)\right) d t, \int_{t_{k-1}}^{t_{k}} \nabla\left(u-v_{k}^{H}\right) d t\right)+\sum_{\Omega=1}^{n} \sum_{i=1}^{k}\left(\int_{t_{i-1}}^{t_{i}} g\left(v_{i}^{H}\right)-g\left(b\left(\eta_{i}\right)\right) d t, \int_{t_{k-1}}^{t_{k}} \nabla\left(u-v_{k}^{H}\right) d t\right), \\
& \left|I_{3}\right| \leq C(\delta) \sum_{k=1}^{n} \tau \sum_{i=1}^{k} \int_{t_{i-1}}^{t_{i}}\left\|g(\theta)-g\left(b\left(v_{i}^{H}\right)\right)\right\|_{L^{2}(\Omega)}^{2} d t+\delta \sum_{k=1}^{n}\left\|\int_{t_{k-1}}^{t_{k}} \nabla\left(u-v_{k}^{H}\right) d t\right\|_{L^{2}(\Omega)}^{2}+\varepsilon \sum_{k=1}^{n} \tau\left\|v_{k}^{H}\right\|_{L^{2}(\Omega)}^{2} \\
& \leq C(\delta) \sum_{k=1}^{n} \tau \int_{t_{0}}^{t_{k}}\left(\theta-b\left(v_{i}^{H}\right), u-v_{i}^{H}\right) d t+\delta \sum_{k=1}^{n}\left\|\int_{t_{k-1}}^{t_{k}} \nabla\left(u-v_{k}^{H}\right) d t\right\|_{L^{2}(\Omega)}^{2}+\varepsilon^{2} .
\end{aligned}
$$

Similarly, the right hand terms $I_{4}, I_{5}, I_{6}$ can be estimated as the following. Use the fact (2.4), we have

$$
\begin{aligned}
& \left|I_{4}\right| \leq \sum_{k=0}^{n}\left\|\theta_{k}-\eta_{k}\right\|_{L^{2}(\Omega)}\left\|\int_{t_{k-1}}^{t_{k}} e_{u} d t\right\|_{L^{2}(\Omega)} \leq\left(\sum_{k=0}^{n} \tau\left\|\theta_{k}-\eta_{k}\right\|_{L^{2}(\Omega)}^{2}\right)^{\frac{1}{2}}\left\|e_{u}\right\|_{L^{2}\left(t_{0}, t_{n} ; L^{2}(\Omega)\right)} \leq C H^{2}, \\
& \left|I_{5}\right| \leq \delta \sum_{k=1}^{n}\left\|\int_{t_{k-1}}^{t_{k}} \nabla\left(u-v_{k}^{H}\right) d t\right\|_{L^{2}(\Omega)}^{2}+C(\delta)\left\|\nabla e_{u}\right\|_{L^{2}\left(t_{0}, t_{n} ; L^{2}(\Omega)\right)}^{2} \leq \delta \sum_{k=1}^{n}\left\|\int_{t_{k-1}}^{t_{k}} \nabla\left(u-v_{k}^{H}\right) d t\right\|_{L^{2}(\Omega)}^{2}+C(\delta) H^{2}, \\
& \left|I_{6}\right| \leq C \sum_{k=1}^{n} \tau \int_{t_{0}}^{t_{k}}\left(\theta-b\left(v_{i}^{H}\right), u-v_{i}^{H}\right) d t+\left\|\nabla e_{u}\right\|_{L^{2}\left(t_{0}, t_{n} ; L^{2}(\Omega)\right)}^{2}+\varepsilon^{2} \leq C \sum_{k=1}^{n} \tau \int_{t_{0}}^{t_{k}}\left(\theta-b\left(v_{i}^{H}\right), u-v_{i}^{H}\right) d t+C\left(H^{2}+\varepsilon^{2}\right) .
\end{aligned}
$$

Combining all the terms and choosing $\delta$ properly, we have

$$
\begin{aligned}
& \int_{t_{0}}^{t_{n}}\left(\theta-b\left(v_{k}^{H}\right), u-v_{k}^{H}\right) d t+\left\|\sum_{k=1}^{n} \int_{t_{k-1}}^{t_{k}} \nabla\left(u-v_{k}^{H}\right) d t\right\|_{L^{2}(\Omega)}^{2}+\sum_{k=1}^{n}\left\|\int_{t_{k-1}}^{t_{k}} \nabla\left(u-v_{k}^{H}\right) d t\right\|_{L^{2}(\Omega)}^{2} \\
& \leq C \sum_{k=1}^{n} \tau \int_{t_{0}}^{t_{k}}\left(\theta-b\left(v_{i}^{H}\right), u-v_{i}^{H}\right)_{\Omega} d t+C\left(\tau+\varepsilon^{2}+H^{2}\right) .
\end{aligned}
$$


Finally, we use Gronwall inequality to get the conclusion of the Theorem 2.2.

\section{Acknowledgements}

The author is supported by the Fundamental Research Funds for the Central Universities (NO.2013B10114).

\section{References}

[1] Alt, H.W. (1985) Nonsteady Fluid Flow through Porous Media. Applications and Theory, 3, 222-228.

[2] Damlamina, A. (1977) Some Result in the Multiphase Stefan Problem. Communications in Partial Differential Equations, 2, 1017-1044. http://dx.doi.org/10.1080/03605307708820053

[3] van Duijn C.J. and Peletier L.A. (1982) Nonstationary Filtration in Partially Saturated Porous Media. Archive for Rational Mechanics and Analysis, 78, 173-198. http://dx.doi.org/10.1007/BF00250838

[4] Nochetto, R.H. and Verdi, C. (1988) Approximation of Degenerate Parabolic Problems Using Numerical Intergration. SIAM Journal on Numerical Analysis, 25, 784-814. http://dx.doi.org/10.1137/0725046

[5] Alt, H.W. and Luckhaus, S. (1983) Quasilinear Elliptic-Parabolic Differential Equations. Mathematische Zeitschrift, 183, 311-341. http://dx.doi.org/10.1007/BF01176474

[6] Ladyzenskaya O., Solonnikov V. and Ural'ceva N. (1968) Linear and Quasilinear Equations of Parabolic Type. Translations of Mathematical Monographs.

[7] Nochetto R.H. (1987) Error Estimates for Multidimensional Singular Parabolic Problems. Japan Journal of Industrial and Applied Mathematics, 4, 111-138. http://dx.doi.org/10.1007/BF03167758

[8] Pop, I.S. (2002) Error Estimates for a Time Discretization Method for Richards’ Equation. Computers \& Geosciences, 6, 141-160. http://dx.doi.org/10.1023/A:1019936917350

[9] Radu, F., Pop, I.S. and Knabner, P. (2004) Oder of Convergence Estimates for an Euler Implicit Mixed Finite Element Discretization of Richards' Equation. SIAM Journal on Numerical Analysis, 22, 1452-1478. http://dx.doi.org/10.1137/S0036142902405229

[10] Pop, I.S. and Yong, W.A. (1997) A maximum Principle Based Numerical Approach to Porous Medium Equation. Proceedings of the 14th Conference on Scientific Computing, 207-218.

[11] Arbogast, T., Wheeler, M.F. and Zhang, N.Y. (1996) A Nonlinear Mixed Finite Element Method for a Degenerate Parabolic Equation Arising in Flow in Porous Media. SIAM Journal on Numerical Analysis, 33, 1669-1687. http://dx.doi.org/10.1137/S0036142994266728

[12] Jager, W. and Kacur, J. (1995) Solution of Doublely Nonlinear and Degenerate Parabolic Problems by Relaxation Schemes. Mathematical Modelling and Numerical Analysis, 29, 605-627.

[13] Adams, R.A. and Fournier, J.J.F. (2003) Sobolev Spaces. 2nd Edition, Academic Press, New York. 